\title{
Memetic Algorithm for Dynamic Optimization Problems
}

\author{
AL-Khafaji Amen \\ University Of Technology, \\ Baghdad, Iraq
}

\begin{abstract}
Dynamic optimization problems has attracted much attention of researchers. However, due to complexity and uncertainty to solve dynamic optimization problems, it very difficult to find out the optimum solution that could be changed over time. Thus, it is necessary to develop efficient or improved an algorithms to solve dynamic optimization problems. A memetic algorithm that based on local search along with an evolutionary algorithm such as genetic algorithm can be used to tackle dynamic optimization problems. This paper investigates the use of multi-crossover operator that is based on heuristic and arithmetic with GA as well as local search for dynamic optimization problems. The proposed approach utilises solution features in terms of diversity and selection to generate better solution. To evaluate the efficiency and feasibility of the proposed operator, a comparison between the results of this study and the results of different works is conducted through a number of evaluations over dynamic optimization problems with various levels of difficulty. The significant findings emerge from this study are the efficiency of the proposed algorithm in solving dynamic environments when compared with other method.
\end{abstract}

\section{General Terms}

Artificial intelligent, dynamic optimization problems, Evolutionary algorithms.

\section{Keywords}

Arithmetic Crossover Operator, Dynamic optimization problems, Evolutionary algorithms, Genetic algorithm, Heuristic crossover, Local search, Mutation Operators, Memetic algorithm.

\section{INTRODUCTION}

Recently, dynamic optimisation problems attract the researches to explore in this filed. Specially, almost of real world problems are dynamic optimisation ones [1-6]. Indeed, unstable environment conditions could directly influence on the nature of objective function. Consequently, landscape of optimal solution will be changed over time. Thus, there is a need to employ an effective algorithm that handle DOPs. Since, Local search could get stick in local optima problem. Additionally, it provides a single solution during time computation. In another words, Local search suffers from offering a new ideal solution under this changing [7]. Therefore, employing memetic algorithm nested of local search will be more beneficial in term of maintaining diversity of solutions as well as selection under changing through time.

A considerable amount of literature has been published on DOPs. Those approaches had been categorized as follows: using explicit and implicit memory strategies [8]; employing bio inspiration approaches [3]; refining population during run time [9]; Extending the diversity of offspring after detecting a change [10]. The recent studies have been highlighted a special model of EA which called memetic algorithm. MA combines both of EA in order to maintaining offspring diversity and local search approaches for refining population during the evaluation [1].

The objective of the present work paper is to investigate MA that based on EA and LS for addressing DOPs. On the particular context, GA will be based on both hybrid crossover operator and mutation-based hill climbing operator. Hence, refinement individuals variety along with detecting the optimal population during this process. Yet, the individuals will passed to hill climbing approach for evaluated the new generation. The efficiency and effectiveness of our proposed MA for DOPs is experimentally validated by applying a popular dynamic optimisation functions.

The remainder of this paper is organized as follows. In Section 2, our proposed MA approach and will describe in detail. Section 3 presents our experimental findings and the results. Finally, the last section draws the conclusions with some recommendations on relevant future work.

\section{PROPOSED ALGORITHM}

In this section, we investigate an enchantment on memetic algorithm for handling effectively DOPs. Essentially, the proposed MA, which consider as a special type from GA, hybridize both of GA with local search in order to refine the adversity of population. As mention above, since, MA has ability to simulate the nature through employing GA. Yet, GA suffers from introduce an effective selection of population during evaluation phase. Hence, including hill climbing with GA as LS will bring its advantages to maintain evaluation process. Basically, the present work deals with multiple phases during the process as will be shown below:

\subsection{Internalize the Solution Representation of Populations}

In this section, we explore the representation of solution population. Basically, binary representation scheme will be employed in order initialize and generate individuals [11]. The scheme fills each population with size ' 100 ' according to Table 1 of parameters sitting randomly where every single bit in chromosome will represent with ' 1 ' or ' 0 '. As a consequence, the objective function will be used to calculate the fitness of new individuals. The generate population will pass to the next phase as explain below in fig1: 


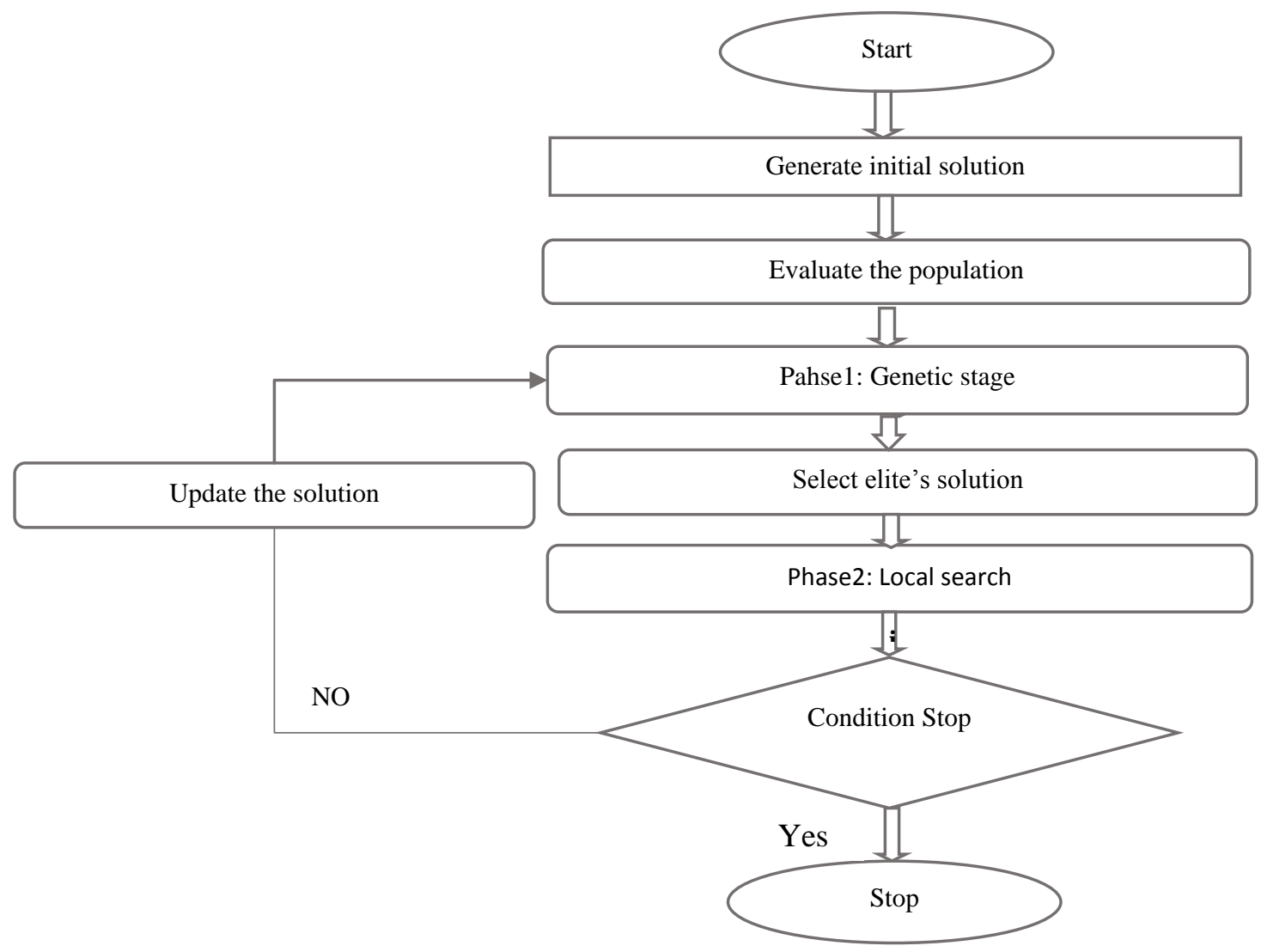

Fig 1: Proposed approach, Memetic Algorithm

\subsection{An Evolutionary Process Phase}

According to Fig1 for population phases, this section of paper deals with GA phase. In General speaking, the performance of GA depend on operates such as crossover and mutation operator [12]. Hence, the proposed MA used a special class of the mention operators for refinement the solutions and their diversity. For more details about those operators with reasons behind use them therefore they will list as follows:

\subsubsection{Hybrid Crossover Operators (HBCO):}

One of most important operator in evolutionary process is crossover operator. Crossover operator hybridize the features of two chromosomes together in order to enhance reproduction chance of population. Generally, crossover operator based on two main factors: Possibility of Crossover (PC) and Cut Point. On the particular context, the performance of crossover operator will be directly relied on selecting an effective cutpoint which will determine the optimal gens. Thus, maintaining diversity of population will be achieved. However, it difficult to detect a worth gens in a chromosome to hybridize them. The proposed MA deals with special type of crossover which based on their fitness. In another words, if one of chromosome was strong while the other ones was inappropriate for mating pool then heuristic crossover operator (HCO) will be applied. HCO flees the characteristics from dump parent indirection away to a worthy individuals. HCO based on the heuristic rule to evaluate chromosome. Whereas, if $\mathrm{P} 1$ and $\mathrm{P} 2$ are population and $\mathrm{P} 1$ was more worthy than $\mathrm{P} 2$ then the new generations will be directly affected by $\mathrm{P} 1$ [13]. $\beta$ which is a tiny value between ' 0 ' and ' 1 'will be initialize randomly according to HCO equation that explain as follows:

Offspring's $=$ Best Parent $+\beta *($ Best Parent - Worst Parent $)$.
On another hand, arithmetic crossover operator (ACO) will employ in case of having identical crossover with same features. ACO based on combine gens of two chromosomes by find mean of them according to the fallowing equation:

Offspring's $=\alpha *$ Best Parent $+(1-\alpha) *$ Worst Parent.

Where $\alpha$ is a constant value set as 0.2 or randomly depend on variants between parents.

\subsubsection{Mutation Operator Based On Hill Climbing:}

Another substantial operator in GA is mutation operator. Specially, in case of crossover operator fails to generate new gens and futures anymore. Mutation operator is about reproduction new gens rather than combine them. Perturbation mechanism is invoked in our proposed MA in order to modify individuals by selecting indiscriminately a gen and complementing it. Thus, the problem of getting stack in local optima will overcame.

\subsection{Hill Climbing Algorithm Phase}

Hill climbing algorithm is employed in this stage. The algorithm start with sitting initial solutions of population, which selected randomly. Later on, the algorithm will iterate the solutions until find the elite's population. Otherwise, it will select the elite chromosomes based on maxim fitness in order to send them for mating pool process according to (possibility of crossover) PC and MP (mutation probability). The result of mating pool will be selected for next iteration until satisfy the stop condition. The result of this phase will represent the elite solution. 


\section{EXPERIMENTSL RESULTS AND COMPARISONS}

In this section, the performance of the proposed memetic algorithm is assessed by employing a most popular binary test functions, which are OneMax, Plateau, Royal Road and Deceptive [14]. Generally speaking, many searchers had been used those functions in order to evaluate the effectiveness of their proposed algorithm. In this article, [1] proposed functions that generate dynamically to adopt with dynamic environments. In Table 1, the parameter of our proposed algorithm are sitting. According to the studies that reported in [1], both of the periodically of changes $\mathrm{T}$ and $\mathrm{P}$ which represent the periodically of changes as well as the amount of change respectively are sitting to prove the efficient of proposed method compare with most modern approaches

Table 1: Parameters settings

\begin{tabular}{|l|l|}
\hline Parameter & Value \\
\hline Population Size & 50 \\
Solution size & 100 \\
Number of iterations & 100 \\
$\mathrm{~T}$ & 100 \\
$\mathrm{P}$ & 0.9 \\
\hline
\end{tabular}

The proposed approach is contrasted with the algorithms that have been mentioned in the literature as follows:

- Pop-HC: An Evolutionary Hill Climbing Algorithm for Dynamic Optimisation Problems [6].

- MIGA: Genetic algorithm with memory based immigrants [15].

- $\quad$ MEGA: memory-enhanced Genetic algorithm [7].

- AHMA: Memetic algorithm with the AHC operator [1].

- MRIGA: Genetic algorithm with memory and random immigrants schemes [15].

Table 2 presents the findings of the proposed algorithm and most modern algorithms.

The efficiency of this work is showed in Table 2. Accordingly, the statistical results reveals the performance of our algorithm performs better that the other algorithms. Even the others did not assesse all test all functions. Thus, the proposed method preserves the diversity during invoking local search.

Table 2: Results Comparison

\begin{tabular}{|l|l|l|l|l|l|l|}
\hline Function name & PMA & Pop-HC & MIGA & MEGA & AHMA & MRIGA \\
\hline OneMax & $\mathbf{8 2 . 4}$ & 86.35 & 94.0 & 79.3 & 95.89 & 80.8 \\
\hline Plateau & $\mathbf{9 9 . 5}$ & 74.21 & - & - & 62.88 & - \\
\hline Royal Road & $\mathbf{9 9 . 7}$ & 51.11 & - & - & 52.52 & - \\
\hline Deceptive & $\mathbf{1 0 0}$ & 72.56 & 71.1 & 83.1 & 85.75 & 68.6 \\
\hline
\end{tabular}

\section{CONCLUSIONS}

A memetic algorithm based on genetic algorithm and hill climbing for dynamic optimization problems is investigated in this paper. A hybrid crossover operators with adaptive hill climbing mutation along with local search had been hybridized by the proposed memetic algorithm. An objective functions of DOPs such as Plateau, Royal Road and Deceptive were used to demonstrate the effectiveness of our method to achieve to refine the adversity of population. The experimental results of this study indicate that our proposed algorithm introduced robust results compared with another methods which had been mentioned in the literature. Further investigation and experimentation into DOPs is strongly recommended. Tthe effects of MA. This research has thrown up many questions in need of further investigation. Further work needs to be done to establish whether the proposed approaches will has same efficacy to sort real DOPs such as Pareto-optimal problem and constructing dynamic optimization problem.

\section{REFERENCES}

[1] Wang, H., D. Wang, and S. Yang, A memetic algorithm with adaptive hill climbing strategy for dynamic optimization problems. Soft Computing, 2009. 13(8-9): p. $763-780$.

[2] Turky, A.M. and S. Abdullah, A multi-population harmony search algorithm with external archive for dynamic optimization problems. Information Sciences, 2014. 272: p. 84-95.

[3] Turky, A.M., S. Abdullah, and N.R. Sabar, A Hybrid Harmony Search Algorithm for Solving Dynamic Optimisation Problems. Procedia Computer Science, 2014. 29: p. 1926-1936.

[4] Turky, A.M. and S. Abdullah, A multi-population electromagnetic algorithm for dynamic optimisation problems. Applied Soft Computing, 2014. 22: p. 474482.

[5] Turky, A.M., S. Abdullah, and N.R. Sabar, Metaheuristic algorithm for binary dynamic optimisation problems and its relevancy to timetabling, in 10th international conference on the Practice and Theory of Automated Timetabling (PATAT 2014), pp. 568-573. 26-29 August 2014, York, UK. 
[6] Turky, A.M., et al., An Evolutionary Hill Climbing Algorithm for Dynamic Optimisation Problems, in The 6th Multidisciplinary Int. conf. On Scheduling: Theory and Applications (MISTA 2013), pp. 795-798. Ghent, Belgium (27-30 Aug 2013).

[7] Yang, S. Memory-based immigrants for genetic algorithms in dynamic environments. in Proceedings of the 7th annual conference on Genetic and evolutionary computation. 2005. ACM.

[8] Talbi, E.-G., A taxonomy of hybrid metaheuristics. Journal of heuristics, 2002. 8(5): p. 541-564.

[9] Yang, S. and X. Yao, Experimental study on populationbased incremental learning algorithms for dynamic optimization problems. Soft Computing, 2005. 9(11): p. 815-834.

[10] Yang, S. Non-stationary problem optimization using the primal-dual genetic algorithm. in Evolutionary Computation, 2003. CEC'03. The 2003 Congress on. 2003. IEEE.
[11] Cobb, H.G., An investigation into the use of hypermutation as an adaptive operator in genetic algorithms having continuous, time-dependent nonstationary environments, 1990, DTIC Document.

[12] Branke, J., et al., A multi-population approach to dynamic optimization problems, in Evolutionary Design and Manufacture2000, Springer. p. 299-307.

[13] Yang, S., Genetic algorithms with elitism-based immigrants for changing optimization problems, in Applications of Evolutionary Computing2007, Springer. p. $627-636$

[14] Grefenstette, J.J. Genetic algorithms for changing environments. in PPSN. 1992.

[15] Yang, S., Genetic algorithms with memory-and elitismbased immigrants in dynamic environments. Evolutionary Computation, 2008. 16(3): p. 385-416. 